\title{
On Cournot-Nash Equilibria with Exogenous Uncertainty ${ }^{1}$
}

\author{
J. AAFTINK, ${ }^{2}$ N. IrELAND, ${ }^{3}$ AND M. SERTEL ${ }^{4}$ \\ Communicated by G. Leitmann
}

\begin{abstract}
A large body of literature has accumulated which examines how the optimal solution of an agent maximizing the expectation of a real-valued function, depending on a random parameter $p$ and the agent's behavior $x$, reacts to perturbations in the first and second moments of $p$. Here, by an approximation valid for small uncertainty, we allow many agents and consider their behavior in a Cournot-Nash equilibrium. We also allow $p$ to depend on the behaviors of the participating agents. We apply the analysis to two models, one of a Cournot oligopoly, the other of a cooperative of individuals where there is uncertainty in the return to communal work.
\end{abstract}

Key Words. Noncooperative games, Cournot-Nash equilibrium, parameter uncertainty, oligopoly, collective farm.

\section{Introduction}

The effects of uncertainty on the optimal decisions of economic agents constitute a topic of considerable importance but commensurate difficulty. The approach of Rothschild and Stiglitz (Refs. 1-2) finds sufficient conditions for determining particular qualitative effects of uncertainty or increased uncertainty. There, sufficient conditions often involve third derivatives of agents' utility functions, and complexities in interpretation often prevent satisfactory results being obtained. Even if reasonable convincing conclusions can be reached, as in the theory of the competitive firm [see also Sandmo (Ref. 3) and Ishii (Ref. 4)], extensions to cases where the dimension

\footnotetext{
${ }^{1}$ The second and third authors are grateful to the British Council (Academic Links and Interchange Scheme) for financial support.

${ }^{2}$ Research Fellow, Department of Applied Mathematics, Twente University of Technology, Enschede, Holland.

${ }^{3}$ Reader in Economics, Department of Economics, University of Warwick, Warwick, England.

${ }^{4}$ Professor, Department of Economics, Bogazici University, Istanbul, Turkey.
} 
of agents' decisions is more than one, or where the outcome to be determined is the result of a number of agents' decisions under uncertainty, are not generally productive.

One alternative approach [Ireland (Ref. 5)], which has a restricted validity, but otherwise appears to offer promise of more fruitful results, is to consider the effects on an agent's behavior of uncertainty in a parameter in relation to the effects on that agent's behavior of a change in the same parameter under certainty. Such an approach uses an approximation which holds better the smaller the amount of uncertainty, and so resulting conditions are in essence necessary conditions for determining qualitative effects of uncertainty. Although not sufficient conditions (for more than small uncertainty) and therefore not implying perfectly general results, they do have two interesting and useful properties. Firstly, they indicate likely effects of uncertainty-and they definitely hold for sufficient small uncertaintyand secondly they allow us to discard the complementary set of outcomes as general qualitative predictions. Thus, if the prediction from our approximation is that less of commodity $i$ will be produced under uncertainty than under certainty, we conclude firstly that this is a likely general result and secondly that more to be produced under uncertainty does not always occur.

The restrictions involved in applying this approximation approach are that the utility of each economic agent is a function (or can be expressed as a function) of a single argument which is linear in the uncertain parameter(s) and strictly concave in the variable(s) over which the individual maximizes the expected value of his or her utility. Thus, for instance, a profit-maximizing price-taking competitive firm decides the quantity $x$ it will produce, at cost $c(x)$, prior to a random price $p$ being revealed. The firm's decision-maker has a subjective probability distribution over $p$ derived from his knowledge of fluctuations in demand. The associated expected value and finite variance of $p$ are $\bar{p}$ and $\sigma^{2}$, respectively. Profit is $p x-c(x)$ and is linear in $p$. If utility is a strictly concave function of profit, due to the decision-maker's risk aversion, then Ireland (Ref. 6) demonstrates that expected utility maximizing behavior is the same as utility maximizing with a nonrisky price lower than $\bar{p}$.

A number of other applications to the theory of the firm satisfy the same formal restrictions [see Ireland (Ref. 6)]. In the present paper, we extend the approach to consider a game among a number of agents where there is exogenous uncertainty concerning one or more parameters. An equilibrium then exists when all agents have determined their behavior prior to the uncertain parameter being revealed.

In Section 2 below, we set out a formal model of $m$ economic agents taking part in a game. We consider a Cournot-Nash equilibrium of agents' behavior in the presence of uncertainty concerning one or more parameters 
in Section 3, and then present two illustrative applications of the analysis in Section 4. Conclusions and some remaining comments are in a final section. The more tedious mathematics are relegated to an Appendix.

\section{A Formal Statement of the Model}

We consider a game $\Gamma$ with a set $M=\{1, \ldots, m\}$ of players indexed by $i$, each equipped with a behavior space $X_{i}$. We define

$$
X^{i}=\prod_{j \in M /\{i\}} X_{j}, \quad X=\prod_{i \in M} X_{i},
$$

denoting generic elements of $X_{i}, X^{i}, X$ by $x_{i}, x^{i}, x=\left(x_{i}, x^{i}\right)$, respectively, $i \in M$. Each player $i \in M$ is assumed to have a utility function $U_{i}: X \rightarrow R$ with a special form. In fact, for each $i \in M$, we posit functions $f_{i}: X \rightarrow R$ and $g_{i}: X \rightarrow R^{n}$, with $g_{i}(x)=\left(g_{i 1}(x), \ldots, g_{i n}(x)\right)^{T}$, and we set

$$
U_{i}(x)=u_{i}\left(y_{i}(x)\right),
$$

where

$$
y_{i}(x)=f_{i}(x)+[p(x)]^{T} g_{i}(x) .
$$

The function $u_{i}: R \rightarrow R$ is assumed to be strictly increasing in $y_{i}$.

Here, $p(x)=\left(p_{1}(x), \ldots, p_{n}(x)\right)^{T} \in R^{n}$, for each $x \in X$, and $p(x)$ is a random vector which has the particular form

$$
p(x)=\bar{p}(x)+\alpha\{r+S \psi(x)\},
$$

where

$$
\bar{p}(x)=a+B \psi(x)
$$

with $a=\left(a_{1}, \ldots, a_{n}\right)^{T} \in R^{n}, B$ an $n \times n$ diagonal matrix whose diagonal is $b=\left(b_{1}, \ldots, b_{n}\right)^{T} \in R^{n}, \psi: X \rightarrow R^{n}$ a function $\left(\psi(x)=\left(\psi_{1}(x), \ldots, \psi_{n}(x)\right)^{T}\right)$, $\alpha \in R, r=\left(r_{1}, \ldots, r_{n}\right)^{T}$ a random vector, and $S$ an $n \times n$ diagonal matrix with diagonal $s=\left(s_{1}, \ldots, s_{n}\right)^{T}$ of random variables. The means of $r$ and $s$ are given by $E\{r\}=E\{s\}=0$, and we denote the covariance matrices $E\left\{r r^{T}\right\}$ by $V$ and $E\left\{s s^{T}\right\}$ by $W$, respectively. Furthermore, $r$ and $s$ are assumed to be independent random vectors, i.e.,

$$
E\left\{r_{i} s_{j}\right\}=0, \quad i, j=1, \ldots, n .
$$

We assume, for each $i \in M$, that $X_{i}$ is an $n_{i}$-dimensional Euclidean space, $n_{i} \geq n$, and that the functions $u_{i}, f_{i}, g_{i}$ are twice continuously differentiable, as is the function $\psi$.

Each agent $i \in M$ is understood to maximize the expected utility $E\left\{u_{i}\right\}$ over $x_{i} \in X_{i}$. 
With these basic data, we will study solutions $x(a, b, \alpha)$ of the game $\Gamma$ according to a Cournot-Nash (noncooperative) solution. the solution $\underline{x}=\underline{x}(a, b, \alpha)$ is assumed to be locally unique within some neighborhood for all possible (relevant) values of $(a, b, \alpha)$. In each case, our interest is in comparing the effect of $(a, b)$ on $x$ with that of $\alpha$. In the style of Ireland (Ref. 6), where nevertheless $p$ is independent of $x$ and $M=\{1\}$, we are able to study (locally) the relations between

$$
\underline{\tilde{x}}(\alpha)-\underline{\hat{x}}
$$

on the one hand, and

$$
\partial \underline{x}(\tilde{a}, b) /\left.\partial a\right|_{a=\tilde{a}} \text { and } \partial \underline{x}(a, \tilde{b}) /\left.\partial b\right|_{b=\tilde{b}}
$$

on the other, where $\stackrel{x}{x}(a, b)=x(a, b, 0)$ denotes the associated solution in the certainty case where $\alpha=0, \tilde{x}(\alpha)=x(\tilde{a}, \tilde{b}, \alpha)$ denotes the associated solution in the case where $(a, b)$ is held fixed to $(\tilde{a}, \tilde{b})$, and $\hat{x}=x(\tilde{a}, \tilde{b}, 0)$.

In the case of a single agent $(m=1)$, the vector $p(x)$ can be interpreted as a vector of prices dependent on the agent's decisions $x$, which may be employment levels of factors of production, amounts of commodities to be produced, marketing decisions, etc. The agent may have market power, i.e., prices dependent on the agent's behavior, in the way defined above in (3) and (4). Then, $\tilde{x}(\alpha)-\hat{x}$ is the effect of uncertainty of magnitude $\alpha$ on optimal decisions and $\partial \underline{\dot{x}}(a, \tilde{b}) / \partial a$ evaluated at $a=\tilde{a}$ and $\partial \underline{x}(\tilde{a}, \tilde{b}) / \partial b$ evaluated at $b=\tilde{b}$ are the changes in optimal decisions given $\alpha=0$ (certainty) of changes in $a$ or $b$ evaluated at $(\tilde{a}, \tilde{b})$. The effect of uncertainty on decisions is thus to be related to the effects of changes in $\vec{p}(x)$ due to changes in $a$ or $b$. Thus, for instance, the effects of uncertainty concerning the intercept and slope of a linear price function $p(x)$ would be related to the effect of parametric changes in such a price function under certainty.

In the case of a number of agents, $m>1$, agent $i$ has control over the variables

$$
x_{i}=\left(x_{i 1}, \ldots, x_{i n}, x_{i, n+1}, \ldots, x_{i n_{i}}\right) .
$$

The variables $\left(x_{i, n+1}, \ldots, x_{i n_{i}}\right)$ are supposed to have no influence on the other agents, i.e., we assume from the beginning that

$$
\partial f_{j} / \partial x_{i k} \equiv 0 \quad \text { and } \quad \partial g_{j} / \partial x_{i k} \equiv 0, \quad k=n+1, \ldots, n_{i}, j \neq i .
$$

An equilibrium is found as the Cournot-Nash noncooperative solution to the $m$-person game, where each agent $i$ is maximizing his or her expected utility given $x^{i}$, that is, the decisions of the other $m-1$ agents. In the Cournot-Nash equilibrium agents are not affected by uncertainty in each other's behavior: this is determined; rather, each agent is influenced by the uncertainty in the $p(x)$ function due to the random vectors $r$ and $s$. If $p(x)$ 
represented a linear inverse industry demand curve where the industry was characterized by a Cournot oligopoly, then although the parameters of demand were uncertain, aggregate supply would be determined at a Cournot-Nash equilibrium where each supplier had no wish to change his output plan given other firms' outputs, and his probability distribution over $r$ and $s$. Note that the $p(x)$ function is independent of $i$-all firms sell at the same price-but that the weight $g_{i}(x)$, in this example output of the $i$ th firm, may be agent specific. If $p(x)$ is a vector of functions, then some elements may not affect all agents and some may only affect a single agent.

\section{Approximation}

The solution $x=x(a, b, \alpha)$ that we examine here is obtained by

$$
\max _{x_{i} \in X_{i}} E\left\{u_{i}\left(y_{i}\left(x_{i}, x^{i}\right)\right)\right\}, \quad i=1, \ldots, m
$$

First-order conditions give

$$
\left.\left(\partial / \partial x_{i}\right) E\left\{u_{i}\left(y_{i}\left(x_{i}, \underline{x}^{i}\right)\right)\right\}\right|_{x_{i}=\underline{x}_{i}}=0, \quad i=1, \ldots, m
$$

In order to compare (5) and (6), we examine (8) when $(a, b)=(\tilde{a}, \tilde{b})$ and obtain the implicit form

$$
F(\underline{\tilde{x}}, \alpha)=-\alpha G(\tilde{\tilde{x}}, \alpha)
$$

where (see Appendix)

$F, G: R^{\sum_{i \in M^{n}} n_{i}} \times R \rightarrow R^{\sum_{i \in M M_{i}} n_{i}}$.

Linear approximation of $F$ and $G$ around $(\tilde{x}, \alpha)=(\underline{x}, 0)$ gives

$$
\begin{aligned}
\frac{\partial}{\partial x}\left[\frac{\partial h_{i}}{\partial x_{i}}(\underline{\hat{x}})\right]^{T}(\underline{\tilde{x}}-\hat{\hat{x}}) & =\alpha^{2} \rho_{i}\left(h_{i}(\hat{\hat{x}})\right)\left[\frac{\partial g_{i}}{\partial x_{i}}(\hat{\hat{x}})\right]^{T} V g_{i}(\underline{\hat{x}}) \\
& +\alpha^{2} \rho_{i}\left(h_{i}(\underline{\hat{x}})\right)\left\{\left[\frac{\partial \psi}{\partial x_{i}}(\underline{\hat{x}})\right]^{T} \operatorname{diag}\left(g_{i}(\hat{\hat{x}})\right)\right. \\
& \left.+\left[\frac{\partial g_{i}}{\partial x_{i}}(\underline{\hat{x}})\right]^{T} \operatorname{diag}(\psi(\underline{\hat{x}}))\right\} W g_{i}^{\psi}(\underline{\hat{x}}), i=1, \ldots, m,
\end{aligned}
$$

where

$$
\rho_{i}\left(y_{i}\right)=-u_{i}^{\prime \prime}\left(y_{i}\right) / u_{i}^{\prime}\left(y_{i}\right),
$$


the coefficient of absolute risk aversion for the $i$ th agent,

$$
\begin{aligned}
& h_{i}(x)=f_{i}(x)+[\bar{p}(x)]^{T} g_{i}(x), \\
& g_{i}^{\psi}(x)=\left[\begin{array}{cc}
g_{i 1}(x) & \psi_{1}(x) \\
\vdots & \vdots \\
g_{i n}(x) & \psi_{n}(x)
\end{array}\right] \text {, }
\end{aligned}
$$

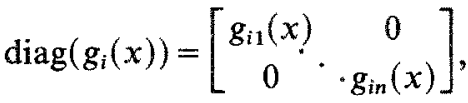

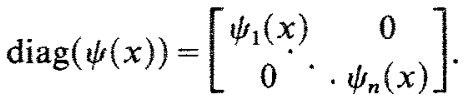

To find

$$
\partial \underline{x}(a, \tilde{b}) /\left.\partial a\right|_{a=\tilde{a}} \text { and } \partial \underline{x}(\tilde{a}, b) /\left.\partial b\right|_{b=\tilde{b}},
$$

we set $\alpha=0$ in (8) and implicity differentiate with respect to $a$ and $b$ to obtain (10a) and (10b) (see Appendix):

$$
\begin{aligned}
& (\tilde{\partial} / \partial x)\left[\frac{\partial h_{i}}{\partial x_{i}}(\hat{\underline{x}})\right]^{T} \frac{\partial \underline{x}}{\partial a}(\tilde{a}, \tilde{b})+\left[\frac{\partial g_{i}}{\partial x_{i}}(\hat{\underline{x}})\right]^{T}=0, \\
& (\partial / \partial x)\left[\frac{\partial h_{i}}{\partial x_{i}}(\underline{\hat{x}})\right]^{T} \frac{\partial \underline{\underline{x}}}{\partial b}(\tilde{a}, \tilde{b})+\left[\frac{\partial \psi}{\partial x_{i}}(\hat{x})\right]^{T} \operatorname{diag}\left(g_{i}(\hat{\hat{x}})\right) \\
& +\left[\frac{\partial g_{i}}{\partial x_{i}}(\hat{\underline{x}})\right]^{T} \operatorname{diag}(\psi(\hat{x}))=0, \quad i=1, \ldots, m .
\end{aligned}
$$

Using (10a) and (10b) in (9) now gives the summary comparison we seek:

$$
\begin{aligned}
& (\partial / \partial x)\left[\frac{\partial h_{i}}{\partial x_{i}}(\hat{x})\right]^{T}(\underline{\tilde{x}}-\underline{\hat{x}})=-\alpha^{2} \hat{\rho}_{i}(\partial / \partial x)\left[\frac{\partial h_{i}}{\partial x_{i}}(\underline{\hat{x}})\right]^{T} \\
& \times\left\{\frac{\partial \underline{\underline{x}}}{\partial a}(\tilde{a}, \tilde{b}) V g_{i}(\hat{x})+\frac{\partial \underline{\underline{x}}}{\partial b}(\tilde{a}, \tilde{b}) W g_{i}^{\psi}(\underline{\hat{x}})\right\}, \quad i=1, \ldots, m,
\end{aligned}
$$

where

$$
\hat{\rho}_{i}=\rho_{i}\left(h_{i}(\underline{\hat{x}})\right) .
$$

Equation (11) is the major result of this paper. It is a very general result relating to a set $M=\{1, \ldots, m\}$ of agents, each making many decisions and faced with $n$ prices or other parameter functions each with two kinds of uncertainty ( $r$ and $s$ ). A general interpretation of (11) will not be attempted, but rather we present in the next section a number of examples and applications which provide useful insights concerning the nature of a Cournot-Nash equilibrium under exogenous uncertainty. In some of the 
applications, we focus on an average or aggregate measure of decisions, rather than individual level decisions. We also consider the case of all identical agents (at least in their behavior under certainty, although they may have different attitudes to risk) and of just two agents. These kinds of assumptions are widely used in discussions of Cournot-Nash equilibria in the existing literature.

\section{Examples and Applications}

4.1. Cournot Oligopoly. The model described in Section 2 has an obvious application in oligopoly theory. Consider $m$ firms each producing an identical product, the industry demand for which is represented by the stochastic inverse demand function $(n=1)$

$$
p(x)=a-b Q+\alpha(r-s Q),
$$

where

$$
Q=\sum_{i=1}^{m} x_{i}
$$

and $(r, s)$ are stochastic. Each agent has the same subjective probability density function on $r$, and similarly on $s$. Random $r$ implies uncertain intercept, and random $s$ implies uncertain slope of the inverse demand function.

Each firm maximizes the (expected) utility from profit, so that the $i$ th firm chooses $x_{i}$ given (true in equilibrium) assumptions about $\underline{x}^{i}$, ex ante of $(r, s)$ being revealed. Profit for the $i$ th firm is

$$
y_{i}(x)=(a-b Q) x_{i}-c_{i}\left(x_{i}\right)+\alpha(r-s Q) x_{i},
$$

so that

$$
h_{i}(x)=-c_{i}\left(x_{i}\right)+\tilde{p}(x) x_{i},
$$

and

$$
(\partial / \partial x)\left[\frac{\partial h_{i}}{\partial x_{i}}(\underline{\hat{x}})\right]=-\left[\tilde{b}, \ldots, \tilde{b}, \tilde{\phi}_{i}+\tilde{b}, \tilde{b}, \ldots, \tilde{b}\right],
$$

where $\tilde{\phi}_{i}$ in the $i$ th component of the $m$-vector,

$$
\tilde{\phi}_{i}=c_{i}^{\prime \prime}\left(\hat{x}_{i}\right)+\tilde{b}
$$

Note that we have assumed no local decisions, i.e.,

$$
n_{i}=n, \quad \text { for all } i \in M \text {. }
$$


Applying the analysis of Section 3 then leads to the summary comparison (11) being of the simpler form,

$$
\begin{aligned}
& {\left[\tilde{b}, \ldots, \tilde{b}, \tilde{\phi}_{i}+\tilde{b}, \ldots, \tilde{b}\right](\underline{\tilde{x}}-\hat{\underline{x}})} \\
& =\alpha^{2} \hat{\rho}_{i} V \underline{\hat{x}}_{i}\left[\tilde{b}, \ldots, \tilde{b}, \tilde{\phi}_{i}+\tilde{b}, \tilde{b}, \ldots, \tilde{b}\right](\partial \underline{\dot{x}} / \partial a) \\
& +\alpha^{2} \hat{\rho}_{i} W \hat{Q} \hat{\underline{x}}_{i}\left[\tilde{b}, \ldots, \tilde{b}, \tilde{\phi}_{i}+\tilde{b}, \tilde{b}, \ldots, \tilde{b}\right](\partial \underline{\dot{x}} / \partial b), \quad i=1, \ldots, m .
\end{aligned}
$$

Equation (16) holds for all $i$, and is useful in a number of particular cases.

Case (i). Firms are identical under certainty, but have different attitudes towards risk [i.e., $y_{i}(\cdot)=y_{j}(\cdot)$, all $i, j$, but different utility functions]. Then, adding Eq. (16) over all $i$ yields, using $\hat{\underline{x}}_{i}=\hat{\underline{x}}_{j}$, all $i, j$ so that $\hat{\underline{x}}_{i}=\hat{Q} / m$ (but $\underline{\underline{x}}_{i}$ is not necessarily equal to $\tilde{x}_{j}$ ), and $\tilde{\phi}_{i}=\tilde{\phi}$, all $i$, we have

$$
\begin{aligned}
(m \tilde{b}+\phi)(\underline{\tilde{Q}}-\underline{\hat{Q}}) & =-\alpha^{2} V(m \tilde{b}+\tilde{\phi})(\underline{\hat{Q}} / m)(\partial \underline{\hat{Q}} / \partial a) \sum_{i=1}^{m}\left(\hat{\rho}_{i} / m\right) \\
& +\alpha^{2} W \underline{\hat{Q}}(m \tilde{b}+\tilde{\phi})(\underline{\hat{Q}} / m)(\partial \underline{Q} / \partial b) \sum_{i=1}^{m}\left(\hat{\rho}_{i} / m\right),
\end{aligned}
$$

or

$$
\tilde{Q}-\hat{Q}=-\alpha^{2}(V \partial \underline{Q} / \partial a-W \underline{\hat{Q}}(\partial \underline{Q} / \partial b))(\underline{\hat{Q}} / m) \sum_{i=1}^{m}\left(\hat{\rho}_{i} / m\right) .
$$

Thus, the proportionate reduction in industry output due to uncertainty in the intercept of the inverse demand function alone $(W=0)$ is

$$
(\tilde{Q}-\underline{Q}) / \underline{\hat{Q}}=-\alpha^{2} V(\partial \underline{Q} / \partial a)(1 / m) \sum_{i=1}^{m}\left(\hat{\rho}_{i} / m\right),
$$

and this depends on the amount of uncertainty $\alpha^{2} V$, the response of industry output defined by a Cournot equilibrium to a parallel shift under certainty $\partial Q / \partial a$, and the average coefficient of absolute risk aversion $\sum_{i=1}^{m}\left(\hat{\rho}_{i} / m\right)$. We would expect $\partial \underline{Q} / \partial a>0$, and so, if on average there is risk aversion, $\left.\sum_{i=1}^{m}\left(\hat{\rho}_{i} / m\right)>0\right)$, then industry output will be less under uncertainty. Uncertainty concerning the intercept of the inverse demand function thus involves an industry output reduction analogous to that for a reduction in the intercept under certainty.

Note that, if uncertainty is involved only in the slope of the inverse demand function, then, for an (on average) risk-averse industry, such uncertainty produces a qualitatively similar response to an increase in $b$ under certainty. An increase in $b$, like a reduction in $a$, produces lower profits and less utility. 
Case (ii). Suppose that $c_{i}^{\prime \prime}\left(\hat{x}_{i}\right)=\hat{c}$, all $i$, i.e., $\tilde{\phi}_{i}=\tilde{\phi}$, all $i$, and $\hat{\rho}_{i} \hat{x}_{i}=\hat{\theta}$, all $i$. In this case, it is no longer assumed that outputs and responses to parameter changes under certainty will be the same for all $i$. Nevertheless, again add (16) over all $i$ and obtain

$$
(m \tilde{b}+\tilde{\phi})(\underline{\hat{Q}}-\hat{Q})=-\alpha^{2} \hat{\theta} V(m \tilde{b}+\tilde{\phi})(\partial \underline{Q} / \partial a)+\alpha^{2} \hat{\theta} W \underline{Q}(m \tilde{b}+\tilde{\phi})(\partial \underline{Q} / \partial b)
$$

or

$$
\tilde{Q}-\hat{Q}=-\alpha^{2} \hat{\theta}[V(\partial \underline{Q} / \partial a)-W \underline{Q}(\partial \underline{Q} / \partial b)],
$$

which is a result comparable with (18), so that the same interpretation of response to risk can be made if firms are not identical under certainty, but have the same slope of marginal cost function $c$ and the same outputweighted coefficient of absolute risk aversion.

Case (iii). Suppose that we have only two firms, $m=2$. Define

$$
\hat{c}_{1}=c_{1}^{\prime \prime}(\underline{\hat{x}})+2 \tilde{b} \text { and } \hat{c}_{2}=c_{2}^{\prime \prime}\left(\underline{\hat{x}}_{2}\right)+2 \tilde{b},
$$

and assume as in Case (ii) that

$$
\hat{\rho}_{1} \hat{x}_{1}=\hat{\rho}_{2} \hat{x}_{2}=\hat{\theta} \text {. }
$$

Then, the formulas for $\underline{\tilde{x}}_{1}-\hat{\underline{x}}_{1}$ and $\underline{\underline{x}}_{2}-\underline{\hat{x}}_{2}$ become very much simpler, even though $\hat{c}_{1}$ is not necessarily equal to $\hat{c}_{2}$,

$$
\underline{\underline{x}}_{i}-\underline{\hat{x}}_{i}=-\alpha^{2} V \hat{\theta}\left(\partial \underline{x}_{i} / \partial a\right)+\alpha^{2} W\left(\underline{\hat{x}}_{1}+\underline{\hat{x}}_{2}\right)\left(\partial \dot{x}_{i} / \partial b\right), \quad i=1,2 .
$$

Again, the same interpretations are possible as in Cases (i) and (ii), only here behavior relates to a particular firm, rather than the industry as a whole.

Alternatively, assume that, as in Case (i), both firms are identical under certainty, so that

$$
\hat{c}_{1}=\hat{c}_{2}=\hat{c} ;
$$

only their attitudes towards risk differ. Then,

$$
\underline{\tilde{x}}_{1}-\underline{\tilde{x}}_{2}=-\alpha^{2}[(\hat{c}+\tilde{b}) /(\hat{c}-\tilde{b})]\left(\hat{\rho}_{1}-\hat{\rho}_{2}\right)\left[V\left(\partial \underline{x}_{i} / \partial a\right)-W Q\left(\partial \underline{x}_{i} / \partial b\right)\right] \underline{\hat{x}}_{i},
$$

so that, assuming

$$
V\left(\partial \underline{x}_{i} / \partial a\right)-W \hat{Q}\left(\partial \underline{x}_{i} / \partial b\right)>0 \text { and }(\hat{c}+\tilde{b}) /(\hat{c}-\tilde{b})>0,
$$

we have that the smaller output under uncertainty is produced by the firm with the higher coefficient of absolute risk aversion. 
4.2. Collective Firms and Private Plots. The application in Section 4.1 only considered one decision variable per agent $\left(n_{i}=n=1\right.$, all $\left.i\right)$, and yet one major aspiration of the procedure outlined in Section 3 was to allow consideration of multiple decision variables. Here, we will show this possibility of applying the analysis to a simple model of a collective farm where the individual worker $i \in\{1, \ldots, m\}$ chooses the allocation of his time to work on the communal land ( $k_{i}$ hours), work on his private plot of land ( $l_{i}$ hours) and leisure [( $\left.E-k_{i}-l\right)$ hours]. For a discussion of collective farms, see Bonin (Ref. 7), Ireland and Law (Ref. 8), Oi and Clayton (Ref. 9), and Sertel (Ref. 10), where the last of these references purports a different analysis, understanding workers to be partners. The utility of an individual member is given by (1), where (2) is of the form

$$
y_{i}=p[q(K) / m]+z_{i}\left(l_{i}\right)-\beta_{i}\left(k_{i}+l_{i}\right),
$$

and $p=\bar{p}+\alpha r$.

We write

$$
K=\sum_{i=1}^{m} k_{i}, \quad L=\sum_{i=1}^{m} l_{i},
$$

so that $q(K)$ is total output of the communal plot which is sold to the state at a price $p$ per unit and then the revenue is distributed equally among all workers on the collective farm. The individual obtains an incomeequivalent return of $z_{i}\left(l_{i}\right)$ from the private plot, and $-\beta_{i}\left(k_{i}+l_{i}\right)$ represents the cost in terms of leisure of working on both the communal and private plots. Nonnegativity conditions on $k_{i}, l_{i}, E-k_{i}-l_{i}$ are assumed to be satisfied at all interesting equilibria. The form of (23) is rather special, but others can be found which still satisfy (2). Now,

$$
\begin{aligned}
& h_{i}\left(k_{1}, l_{1}, k_{2}, l_{2}\right)=\bar{p}[q(K) / m]+z_{i}\left(l_{i}\right)-\beta_{i}\left(k_{i}+l_{i}\right), \\
& g_{i}\left(k_{1}, l_{1}, k_{2}, l_{2}\right)=q(K) / m,
\end{aligned}
$$

so that, for just two individuals $(m=2)$, the left-hand-side of Eq. (11), stacked for $i=1,2$, is

$$
\left[\begin{array}{cccc}
\bar{p} q^{\prime \prime} / 2-\beta_{1}^{\prime \prime} & -\beta_{1}^{\prime \prime} & \bar{p} q^{\prime \prime} / 2 & 0 \\
-\beta_{1}^{\prime \prime} & \left(z_{1}^{\prime \prime}-\beta_{1}^{\prime \prime}\right) & 0 & 0 \\
\bar{p} q^{\prime \prime} / 2 & 0 & \bar{p} q^{\prime \prime} / 2-\beta_{2}^{\prime \prime} & -\beta_{2}^{\prime \prime} \\
0 & 0 & -\beta_{2}^{\prime \prime} & \left(z_{2}^{\prime \prime}-\beta_{2}^{\prime \prime}\right)
\end{array}\right]\left[\begin{array}{c}
\tilde{k}_{1}-\hat{k}_{1} \\
\tilde{l}_{1}-\hat{\underline{l}}_{1} \\
\tilde{\hat{k}}_{2}-\hat{\underline{k}}_{2} \\
\tilde{\underline{l}}_{2}-\hat{\underline{l}}_{2}
\end{array}\right],
$$

where the components of the matrix are evaluated at

$$
\left(k_{1}, l_{1}, k_{2}, l_{2}\right)=\left(\hat{k}_{1}, \hat{I}_{1}, \hat{k}_{2}, \hat{l}_{2}\right) \text {. }
$$


Describe the matrix in (25) as $\hat{H}$; then, the right-hand side of the stack of equations (11) is

$$
-\alpha^{2}[q(\hat{K}) / 2] V\left[\begin{array}{cccc}
\hat{\rho}_{1} & & & 0 \\
& \hat{\rho}_{1} & & \\
& & \hat{\rho}_{2} & \\
& 0 & & \hat{\rho}_{2}
\end{array}\right] \hat{H}\left[\begin{array}{l}
d \underline{\underline{k}}_{1} / d \bar{p} \\
d \underline{l}_{1} / d \bar{p} \\
d \underline{k}_{2} / d \bar{p} \\
d \underline{l}_{2} / d \bar{p}
\end{array}\right] .
$$

Now, simple results can be obtained in at least two cases.

Case (i). Let $\hat{\rho}_{1}=\hat{\rho}_{2}=\hat{\rho}$. Then, provided $\hat{H}^{-1}$ exists,

$$
\tilde{\tilde{x}}_{i}-\underline{\hat{x}}_{i}=-\alpha^{2}[q(\underline{\hat{K}}) / 2] V \hat{\rho}\left(d \underline{x}_{i} / d \bar{p}\right), \quad x_{i}=\left(k_{i}, l_{i}\right), \quad i=1,2 .
$$

Thus, for each variable and for each individual, the response to uncertainty in price from communal output is the same constant

$$
\lambda=-\alpha^{2}[q(\underline{\hat{K}}) / 2] V \hat{\rho}
$$

of the respective adjustment to a change in $\bar{p}$.

Case (ii). Now, consider the agents as identical in the certainty situation [same $h(\cdot)$ function], but allow them to have different attitudes to risk $\left(\hat{\rho}_{i}\right)$. Then, (26) can be written as

$$
-\alpha^{2}[q(\hat{\underline{K}}) / 2] V\left[\begin{array}{cccc}
\hat{\rho}_{1} & & & 0 \\
& \hat{\rho}_{1} & & \\
& & \hat{\rho}_{2} & \\
0 & & \hat{\rho}_{2}
\end{array}\right]\left[\begin{array}{ccc}
\bar{p} q^{\prime \prime}-\beta^{\prime \prime} & -\beta^{\prime \prime} \\
-\beta^{\prime \prime} & z^{\prime \prime}-\beta^{\prime \prime} \\
\bar{p} q^{\prime \prime}-\beta^{\prime \prime} & -\beta^{\prime \prime} \\
-\beta^{\prime \prime} & z^{\prime \prime}-\beta^{\prime \prime}
\end{array}\right]\left[\begin{array}{c}
d \underline{\mathscr{K}} / d \bar{p} \\
\\
d \underline{\circ} / d \bar{p}
\end{array}\right],
$$

as

$$
d \underline{\underline{k}}_{1} / d \bar{p}=d \underline{\underline{k}}_{2} / d \bar{p},
$$

etc. Now, add the first and third rows and the second and the last rows of (27) and (28) to obtain

$$
\begin{aligned}
& {\left[\begin{array}{cccc}
\bar{p} q^{\prime \prime}-\beta^{\prime \prime} & -\beta^{\prime \prime} & \bar{p} \underline{q}^{\prime \prime}-\beta^{\prime \prime} & -\beta^{\prime \prime} \\
-\beta^{\prime \prime} & z^{\prime \prime}-\beta^{\prime \prime} & -\beta^{\prime \prime} & z^{\prime \prime}-\beta^{\prime \prime}
\end{array}\right]\left[\begin{array}{c}
\tilde{k}_{1}-\hat{k}_{1} \\
\tilde{l}_{1}-\hat{\underline{l}}_{1} \\
\hat{\hat{k}}_{2}-\hat{\hat{k}}_{2} \\
\tilde{l}_{2}-\underline{\hat{l}}_{2}
\end{array}\right]} \\
& =\left[\begin{array}{cc}
\bar{p} q^{\prime \prime}-\beta^{\prime \prime} & -\beta^{\prime \prime} \\
-\beta^{\prime \prime} & z^{\prime \prime}-\beta^{\prime \prime}
\end{array}\right]\left[\begin{array}{c}
\tilde{\tilde{K}}-\underline{\underline{K}} \\
\tilde{\tilde{L}}-\underline{\hat{L}}
\end{array}\right]
\end{aligned}
$$

from (25), and

$$
-\alpha^{2}[q(\underline{\hat{K}}) / 2] V\left(\hat{\rho}_{1}+\hat{\rho}_{2}\right)\left[\begin{array}{cc}
\bar{p} q^{\prime \prime}-\beta^{\prime \prime} & -\beta^{\prime \prime} \\
-\beta^{\prime \prime} & z^{\prime \prime}-\beta^{\prime \prime}
\end{array}\right]\left[\begin{array}{c}
d \stackrel{\circ}{\underline{K}} / d \bar{p} \\
d \underline{L} / d \bar{p}
\end{array}\right]
$$


from (28). Thus, provided the inverse of

$$
\left[\begin{array}{cc}
\bar{p} q^{\prime \prime}-\beta^{\prime \prime} & -\beta^{\prime \prime} \\
-\beta^{\prime \prime} & z^{\prime \prime}-\beta^{\prime \prime}
\end{array}\right]
$$

exists, we have

$$
\left[\begin{array}{c}
\underline{\tilde{K}}-\hat{\hat{K}} \\
\tilde{\tilde{L}}-\underline{\hat{L}}
\end{array}\right]=-\alpha^{2}[q(\hat{\hat{K}}) / 2] V\left(\hat{\rho}_{1}+\hat{\rho}_{2}\right)\left[\begin{array}{l}
d \underline{\dot{R}} / d \bar{p} \\
d \underline{\dot{o}} / d \bar{p}
\end{array}\right] .
$$

Again, the relationship between $\tilde{K}-\hat{K}$ and $d \underline{K} / d \bar{p}$ is the same as between $\tilde{L}-\hat{L}$ and $d \underline{L} / d \bar{p}$, so that uncertainty in communal product price has an effect on total labor supplies analogous to a reduction in that price under certainty, if individuals are, on average, risk averse.

The application above can be generalized in a number of directions. Particularly, $m>2$ presents no problem. For Case (ii), other distribution rules for communal plot revenue, such as according to individual labor input [see Ireland and Law (Refs. 5 and 11)], and other specifications for (2) can be accommodated with no additional difficulty.

\section{Conclusions and Extensions}

We saw in Section 4 how, by appropriate assumptions and restrictions, Eqs. (11), all $i$, could be used to relate differences in individual or aggregate behavior due to uncertainty in parameter to responses to changes in that parameter under certainty. Consider one such result, Eq. (27). Suppose one posed the question, what difference in parameter value under certainty would produce (approximately) the same behavior as the given amount of uncertainty, i.e., $\Delta p$ which solves

$$
\underline{\hat{x}}_{i}(\alpha)=\underline{\underline{x}}_{i}(\bar{p}+\Delta p) \text {. }
$$

The answer is simply [for the result (27)]

$$
\Delta p=-\alpha^{2}[q(\hat{\hat{K}}) / 2] V \rho,
$$

and $\Delta p$ of opposite sign could be used as a compensation to maintain the same behavior with the onset of uncertainty. Note, however, that this would overcompensate a risk-averse individual in terms of his expected utility. An Arrow-Pratt risk premium, such that utility is equivalent to that under certainty, would be approximately one-half of $\Delta p$ in (30).

We have concentrated our analysis on a Cournot-Nash noncooperative solution. However, other possible solution concepts to the game $\Gamma$ might be applied and can be the subject for further research. One obvious one we should mention here is a cooperative solution. In the case of identical utility functions and cost functions, an oligopoly problem such as in Section 
4.1 reduces to maximizing the expected utility of an average firm, with respect to all decision variables $x\left(x_{i} \equiv x\right)$. Then,

$$
(\partial / \partial x)\left[\frac{\partial h}{\partial x_{i}}(\underline{\hat{x}})\right]^{T}=(\partial / \partial x)\left[\frac{\partial h}{\partial x}(\underline{\hat{x}})\right]^{T}
$$

forms a square nonsingular matrix, and immediately

$$
\underline{\tilde{x}}-\hat{\hat{x}}=-\alpha^{2} \hat{\rho}\left\{(\partial \underline{\dot{x}} / \partial a) V g(\underline{\hat{x}})+(\partial \underline{\dot{x}} / \partial b) W g^{\psi}(\underline{\hat{x}})\right\}
$$

It is likely that most other solution concepts to the game do not yield such tractable results as the Cournot-Nash solution. In particular, a Stackelberg solution appears unlikely to be simplified by the application of the approach we have used here. However, generalizations of the Cournot-Nash solution involving nonzero conjectural variation of other agents' responses to an agent's own change in behavior may well lead to further results. Confirmation of these propositions is a matter for further research.

\section{Appendix}

The Cournot-Nash solution $x(a, b, \alpha)$ to the game $\Gamma$ considered in Section 2 is characterized by the following set of equations:

$$
\left.\left(\partial / \partial x_{i}\right) E\left\{u_{i}\left(y_{i}\left(x_{i}, \underline{x}^{i}\right)\right)\right\}\right|_{x_{i}=x_{i}}=0, \quad i=1, \ldots, m,
$$

or

$$
E u_{i}^{\prime}\left(y_{i}(\underline{x})\right)\left[\partial y_{i}(\underline{x}) / \partial x_{i}\right]=0, \quad i=1, \ldots, m .
$$

By definition of $y_{i}$,

$$
\begin{aligned}
\partial y_{i} / \partial x_{i} & =\partial h_{i} / \partial x_{i}+\alpha r^{T}\left(\partial g_{i} / \partial x_{i}\right)+\alpha\left[g_{i}(x)\right]^{T} S\left(\partial \psi / \partial x_{i}\right) \\
& +\alpha[\psi(x)]^{T} S\left(\partial g_{i} / \partial x_{i}\right)
\end{aligned}
$$

where

$$
h_{i}(x):=f_{i}(x)+a^{T} g_{i}(x)+[\psi(x)]^{T} B g_{i}(x) .
$$

o, (31) becomes

$$
\begin{aligned}
& E\left\{u_{i}^{\prime}\left(y_{i}(x)\right)\right\}\left[\partial h_{i}(x) / \partial x_{i}\right]^{T}+\alpha E\left\{u_{i}^{\prime}\left(y_{i}(\underline{x})\right)\left[\partial g_{i}(\underline{x}) / \partial x_{i}\right]^{T} r\right\} \\
& +\alpha E\left\{u_{i}^{\prime}\left(y_{i}(x)\right)\left[\partial \psi(\underline{x}) / \partial x_{i}\right]^{T} S g_{i}(\underline{x})\right\} \\
& +\alpha E\left\{u_{i}^{\prime}\left(y_{i}(\underline{x})\right)\left[\partial g_{i}(\underline{x}) / \partial x_{i}\right]^{T} S \psi(\underline{x})\right\}=0, \quad i=1, \ldots, m .
\end{aligned}
$$

Now, calculate $\partial \underline{x} / \partial a$ and $\partial \underline{x} / \partial b$, set $\alpha=0$ in (32), and obtain $u_{i}^{\prime}\left(h_{i}(\underline{x})\left[\partial h_{i}(\underline{\dot{x}}) / \partial x_{i}\right]^{T}=0, \quad i=1, \ldots, m\right.$, 
or, since $u_{i}(\cdot)$ is strictly increasing,

$$
\left[\partial h_{i}(\underline{x}(a, b)) / \partial x_{i}\right]^{T}=0, \quad i=1, \ldots, m .
$$

Notice that, from (33), we also have

$$
\left[\partial h_{i}(\underline{\hat{x}}) / \partial x_{i}\right]^{T}=0, \quad i=1, \ldots, m .
$$

Equation (33) written out gives

$$
\begin{aligned}
{\left[\partial f_{i}(\underline{\dot{o}}(a, b)) / \partial x_{i}\right]^{T}+\left[\partial g_{i}(\underline{\dot{x}}(a, b)) / \partial x_{i}\right]^{T} a } \\
\quad+[\psi(\underline{\dot{x}}(a, b))]^{T} B\left[\partial g_{i}(\underline{\dot{x}}(a, b)) / \partial x_{i}\right] \\
\quad+\left[g_{i}(\underline{o}(a, b))\right]^{T} B\left[\partial g(\underline{\dot{x}}(a, b)) / \partial x_{i}\right]=0, \quad i=1, \ldots, m .
\end{aligned}
$$

Differentiation with respect to $a$ and then $b$ of (35) at $(a=\tilde{a}, b=\tilde{b})$, yields (10a) and (10b), respectively. For determining $\underline{\tilde{x}}-\hat{x}$, set $(a, b)=(\tilde{a}, \tilde{b})$ in (32), and obtain

$$
\begin{aligned}
& E\left\{u_{i}^{\prime}\left(y_{i}(\underline{\tilde{x}})\right)\right\}\left[\partial h_{i}(\underline{\tilde{x}}) / \partial x_{i}\right]^{T}=(-\alpha)\left[E\left\{u_{i}^{\prime}\left(y_{i}(\underline{\tilde{x}})\right)\left[\partial g_{i}(\underline{\tilde{x}}) / \partial x_{i}\right]^{T} r\right\}\right. \\
& \quad+E\left\{u_{i}^{\prime}\left(y_{i}(\underline{\tilde{x}})\right)\left[\partial \psi(\underline{\tilde{x}}) / \partial x_{i}\right]^{T} S g_{i}(\underline{\tilde{x}})\right. \\
& \left.\quad+E\left\{u_{i}^{\prime}\left(y_{i}(\underline{\tilde{x}})\right)\left[\partial g_{i}(\underline{\tilde{x}}) / \partial x_{i}\right]^{T} S \psi(\underline{\tilde{x}})\right\}\right], \quad i=1, \ldots, m .
\end{aligned}
$$

Let the left-hand side of (36) be defined as $F_{i}(\tilde{x}, \alpha)$ and the three right-hand side terms inside the square brackets as $G^{l}(\underline{\tilde{x}}, \alpha), l=\mathrm{I}$, II, III, respectively. First, consider

$$
F(\underline{\tilde{x}}, \alpha):=\left[\begin{array}{c}
F_{1}(\underline{\tilde{x}}, \alpha) \\
\vdots \\
F_{m}(\underline{\tilde{x}}, \alpha)
\end{array}\right] .
$$

Linear approximation of $F$ around $(\underline{\tilde{x}}, \alpha)=(\underline{\hat{x}}, 0)$ is given by

$$
F(\underline{\tilde{x}}, \alpha) \approx F(\underline{\tilde{x}}, 0)+[\partial F(\underline{\hat{x}}, 0) / \partial x](\underline{\tilde{x}}-\underline{\hat{x}})+[\partial F(\underline{\hat{x}}, 0) / \partial \alpha] \alpha,
$$

or

$F_{i}(\underline{\tilde{x}}, \alpha)=F_{i}(\underline{\tilde{x}}, 0)+\left[\partial F_{i}(\underline{\hat{x}}, 0) / \partial x\right](\underline{\tilde{x}}-\underline{\hat{x}})+\left[\partial F_{i}(\underline{\hat{x}}, 0) / \partial \alpha\right] \alpha, i=1, \ldots, m$.

Now,

$$
\begin{aligned}
F_{i}(\hat{\hat{x}}, 0)= & u_{i}^{\prime}\left(h_{i}(\underline{\hat{x}})\right)\left[\partial h_{i}(\hat{\hat{x}}) / \partial x_{i}\right]^{T}=0 \\
\frac{\partial F_{i}}{\partial x}(\underline{\tilde{x}}, \alpha) & =\left[\frac{\partial h_{i}}{\partial x_{i}}(\underline{\tilde{x}})\right]^{T} E\left\{u_{i}^{\prime \prime}\left(y_{i}(\underline{\tilde{x}})\right)\left[\frac{\partial y_{i}}{\partial x}(\underline{\tilde{x}})\right]\right\} \\
& +E\left\{u_{i}^{\prime}\left(y_{i}(\underline{\tilde{x}})\right)\right\}(\partial / \partial x)\left[\frac{\partial h_{i}}{\partial x_{i}}(\underline{\hat{x}})\right]^{T}
\end{aligned}
$$




$$
\begin{aligned}
& \frac{\partial F_{i}}{\partial x}(\underline{\tilde{x}}, 0)=u_{i}^{\prime}\left(h_{i}(\hat{\underline{x}})\right)(\partial / \partial x)\left[\frac{\partial h_{i}}{\partial x_{i}}(\underline{\hat{x}})\right]^{T}, \\
& \frac{\partial F_{i}}{\partial \alpha}(\underline{\tilde{x}}, \alpha)=E\left\{u_{i}^{\prime \prime}\left(y_{i}(\tilde{x})\right)\left[\frac{\partial y_{i}}{\partial \alpha}(\tilde{\tilde{x}})\right]\right\}\left[\frac{\partial h_{i}}{\partial x_{i}}(\underline{\tilde{x}})\right]^{T}, \\
& \frac{\partial F_{i}}{\partial \alpha}(\hat{x}, 0)=0,
\end{aligned}
$$

and thus

$$
F_{i}(\underline{\tilde{x}}, \alpha) \approx u_{i}^{\prime}\left(h_{i}(\underline{\hat{x}})\right)(\partial / \partial x)\left[\frac{\partial h_{i}}{\partial x_{i}}(\underline{\hat{x}})\right]^{T}(\underline{\tilde{x}}-\underline{\hat{x}}), \quad i=1, \ldots, m .
$$

Linear approximation of the term in the square brackets on the right-hand side of (36) is the sum of the linear approximations of the three terms in these brackets. We can rewrite $G^{I}(\underline{x}, \alpha)$ as

$$
\begin{aligned}
G_{i}^{\mathrm{I}}(\tilde{\tilde{x}}, \alpha) & =E\left\{u_{i}^{\prime}\left(y_{i}(\underline{\tilde{x}})\right) \sum_{j=1}^{n} r_{j}\left[\partial g_{i j}(\underline{\tilde{x}}) / \partial x_{i}\right]^{T}\right\} \\
& =\sum_{j=1}^{n} E\left\{u_{i}^{\prime}\left(y_{i}(\underline{\tilde{x}})\right) r_{j}\right\}\left[\partial g_{i j}(\underline{\tilde{x}}) / \partial x_{i}\right]^{T}
\end{aligned}
$$

Further,

$$
\begin{aligned}
& G_{i}^{\mathrm{I}}(\hat{\hat{x}}, 0)= \sum_{j=1}^{n} u_{i}^{\prime}\left(h_{i}(\underline{\hat{x}})\right) E\left\{r_{j}\right\}\left[\partial g_{i j}(\underline{\hat{x}}) / \partial x_{i}\right]^{T}=0 \\
& \frac{\partial G_{i}^{\mathrm{I}}}{\partial x}(\underline{\tilde{x}}, \alpha)= \sum_{j=1}^{n}\left[\frac{\partial g_{i j}}{\partial x_{i}}(\underline{\tilde{x}})\right]^{T} E\left\{u_{i}^{\prime \prime}\left(y_{i}(\underline{\tilde{x}})\right)\left[\frac{\partial y_{i}}{\partial x}(\underline{\tilde{x}})\right] r_{j}\right\} \\
&+\sum_{j=1}^{n} E\left\{u_{i}^{\prime}\left(y_{i}(\underline{\tilde{x}})\right) r_{j}\right\}(\partial / \partial x)\left[\frac{\partial g_{i j}}{\partial x_{i}}(\underline{\tilde{x}})\right]^{T}, \\
& \frac{\partial G_{i}^{1}}{\partial x}(\hat{x}, 0)= 0, \\
& \frac{\partial G_{i}^{\mathrm{I}}}{\partial \alpha}(\underline{\tilde{x}}, \alpha)= \sum_{j=1}^{n} E\left\{u_{i}^{\prime \prime}\left(y_{i}(\underline{\tilde{x}})\right)\left[\frac{\partial y_{i}}{\partial \alpha}(\underline{\tilde{x}})\right] r_{j}\right\}\left[\frac{\partial g_{i j}}{\partial x_{i}}(\underline{\tilde{x}})\right]^{T}, \\
& \partial y_{i} / \partial \alpha=r^{T} g_{i}(\underline{\tilde{x}})+[\psi(\underline{\tilde{x}})]^{T} S g_{i}(\underline{\tilde{x}}), \\
& \frac{\partial G_{i}^{1}}{\partial \alpha}(\hat{\hat{x}}, 0)=\sum_{j=1}^{n} u_{i}^{\prime \prime}\left(h_{i}(\underline{\hat{x}})\right) E\left\{r^{T} g_{i}(\hat{x}) r_{j}+[\psi(\underline{\hat{x}})]^{T} S g_{i}(\underline{\hat{x}}) r_{j}\left[\frac{\partial g_{i j}}{\partial x_{i}}(\underline{\hat{x}})\right]^{T} .\right.
\end{aligned}
$$

By definition,

$$
\begin{aligned}
& E\left\{r_{j} r^{T}\right\}=V_{j^{*}}(j \text { th row of } V), \\
& E\left\{r_{j} s_{k}\right\}=0 ;
\end{aligned}
$$


so,

$$
\begin{aligned}
\frac{\partial G_{i}^{I}}{\partial \alpha}(\hat{x}, 0) & =\sum_{j=1}^{n} u_{i}^{\prime \prime}\left(h_{i}(\hat{\underline{x}})\right) V_{j^{*}} g_{i}(\hat{x})\left[\frac{\partial g_{i j}}{\partial x_{i}}(\hat{x})\right]^{T} \\
& =u_{i}^{\prime \prime}\left(h_{i}(\hat{\hat{x}})\right)\left[\frac{\partial g_{i}}{\partial x_{i}}(\hat{\hat{x}})\right]^{T}\left[\begin{array}{c}
V_{1^{*}} g_{i}(\hat{x}) \\
\vdots \\
\left.V_{n^{*}} g_{i}(\hat{\underline{x}})\right]
\end{array}\right. \\
& =u_{i}^{\prime \prime}\left(h_{i}(\hat{\underline{x}})\right)\left[\frac{\partial g_{i}}{\partial x_{i}}(\hat{x})\right]^{T} V g_{i}(\hat{x}) .
\end{aligned}
$$

Therefore,

$$
G_{i}^{\mathrm{I}}(\underline{\tilde{x}}, \alpha) \approx \alpha u_{i}^{\prime \prime}\left(h_{i}(\underline{\hat{x}})\left[\partial g_{i}(\underline{\hat{x}}) / \partial x_{i}\right]^{T} V g_{i}(\underline{\hat{x}}), \quad i=1, \ldots, m .\right.
$$

In a similar way, we can obtain

$$
\begin{aligned}
& G_{i}^{\mathrm{II}}(\underline{\tilde{x}}, \alpha) \approx \alpha u_{i}^{\prime \prime}\left(h_{i}(\underline{\hat{x}})\right)\left[\partial \psi(\hat{\hat{x}}) / \partial x_{i}\right]^{T} \operatorname{diag}\left(g_{i}(\underline{\hat{x}})\right) W \operatorname{diag}\left(g_{i}(\underline{\hat{x}})\right) \psi(\underline{\hat{x}}), \\
& i=1, \ldots, m \text {, } \\
& G_{i}^{\mathrm{III}}(\underline{\tilde{x}}, \alpha) \approx \alpha u_{i}^{\prime \prime}\left(h_{i}(\underline{\hat{x}})\right)\left[\partial g_{i}(\underline{\hat{x}}) / \partial x_{i}\right]^{T} \operatorname{diag}(\psi(\underline{\hat{x}})) W \operatorname{diag}(\psi(\hat{\underline{x}})) g_{i}(\underline{\hat{x}}), \\
& \text { Then, (38)-(42) combined give } \\
& i=1, \ldots, m \text {. }
\end{aligned}
$$

$$
F_{i}=-\alpha\left(G_{i}^{1}+G_{i}^{11}+G_{i}^{111}\right)
$$

which, using (36) and (37), can be simplified to yield Eq. (9). Then, with (10a) and (10b), we have

$$
(\partial / \partial x)\left[\frac{\partial h_{i}}{\partial x_{i}}(\underline{\hat{x}})\right]^{T}(\underline{\hat{x}}-\hat{\hat{x}})=-\alpha^{2} \rho_{i}\left(h_{i}(\hat{x})\right)(\partial / \partial x)\left[\frac{\partial h_{i}}{\partial x_{i}}(\hat{\hat{x}})\right]^{T}
$$

which is Eq. (11).

Note that the dimensions of the terms in (11) are

$$
\begin{array}{ll}
(\partial / \partial x)\left[\frac{\partial h_{i}}{\partial x_{i}}(\underline{\hat{x}})\right]^{T}, & n_{i} \times \sum_{i=1}^{m} n_{i}, \\
\tilde{x}-\hat{\hat{x}}: \sum_{i=1}^{m}, & n_{i} \times 1, \\
\alpha, \rho_{i}\left(h_{i}(\hat{\hat{x}})\right), & 1 \times 1, \\
V, W, & n \times n, \\
g_{i}(\hat{x}), g_{i}^{\psi}(\hat{x}), & n \times 1, \\
\partial \underline{\hat{x}} / \partial a, \partial \underline{\underline{x}} / \partial b, & \sum_{i=1}^{m} n_{i} \times n .
\end{array}
$$




\section{References}

1. RothsCHILD, M., and STIGLITZ, J. E., Increasing Risk, I: A Definition, Journal of Economic Theory, Vol. 2, pp. 225-243, 1970.

2. Rothschild, M., and STIGLITZ, J. E., Increasing Risk, II: Its Economic Consequences, Journal of Economic Theory, Vol. 3, pp. 66 84, 1971.

3. SANDmo, A., On the Theory of the Competitive Firm under Price Uncertainty, American Economic Review, Vol. 61, pp. 65-73, 1971.

4. IsHrI, Y., On the Theory of the Competitive Firm under Price Uncertainty: Note, American Economic Review, Vol. 67, pp. 768-769, 1977.

5. IRELAND, N. J., and LAW, P. J., The Economics of Labor-Managed Enterprises, Croom Helm, London, England, 1982.

6. IreLAND, N. J., The Analogy between Parameter Uncertainty and Parameter Change, Economics Letters, Vol. 6, pp. 301-308, 1980.

7. BONIN, J. P., Work Incentives and Uncertainty on a Collective Farm, Journal of Comparative Economics, Vol. 1, pp. 77-97, 1977.

8. Ireland, N. J., and LAw, P. J., Private Plot Restrictions in a Collective Farm Model, Canadian Journal of Economics, Vol. 13, pp. 475-485, 1980.

9. Oi, W. Y., and Clayton, E. S., A Peasant's View of a Soviet Collective Farm, American Economic Review, Vol. 58, pp. 37-59, 1968.

10. SERTEL, M. R., Workers and Incentives, North-Holland Publishing Company, Amsterdam, Holland, 1982.

11. IRELAND, N. J., and LAW, P. J., Efficiency, Incentives, and Individual Labor Supply in the Labor-Managed Firm, Journal of Comparative Economics, Vol. 5, pp. 1-23, 1981. 